\title{
THE LOCAL SPECTRAL BEHAVIOR OF COMPLETELY SUBNORMAL OPERATORS $\left({ }^{1}\right)$
}

\author{
BY \\ K. F. CLANCEY AND C. R. PUTNAM
}

\begin{abstract}
For any compact set $X$, let $C(X)$ denote the continuous functions on $X$ and $R(X)$ the functions on $X$ which are uniformly approximable by rational functions with poles off $X$. Let $A$ denote a subnormal operator having no reducing space on which it is normal. It is shown that a necessary and sufficient condition that $X$ be the spectrum of such an operator $A$ is that $R(X \cap \bar{D}) \neq C(X \cap \bar{D})$ whenever $D$ is an open disk intersecting $X$ in a nonempty set.
\end{abstract}

1. Introduction. An operator $A$ on a Hilbert space $\mathfrak{S}$ is said to be subnormal if there exists a normal operator $N$ on a Hilbert space $\mathfrak{\Omega} \supset \mathfrak{S}$ with the property that $\mathfrak{F}$ is invariant under $N$ and $A$ is the restriction of $N$ to $\mathfrak{F}$. It was proved by Halmos [5] that if $N$ is the minimal normal extension of $A$ then $\operatorname{sp}(N) \subset \operatorname{sp}(A)$. Bram [1] proved that $\operatorname{sp}(A)$ consists of $\operatorname{sp}(N)$ together with some (possibly all or none) of the holes of $\operatorname{sp}(N)$. (A hole of a compact set is a bounded component of its complement.) An easy proof of this due to S. K. Parrott is given in Halmos [6, p. 310].

An operator $T$ on a Hilbert space $\mathfrak{S}$ is said to be hyponormal if $T^{*} T-T T^{*} \geqq 0$ and completely hyponormal if, in addition, there exists no nontrivial reducing subspace of $T$ on which $T$ is normal. It is known that the subnormal operators constitute a proper subset of the hyponormal ones. A subnormal operator $A$ will be called completely subnormal if it is completely hyponormal.

A compact set $X$ of the complex plane is called a spectral set for an operator $T$ on a Hilbert space $\mathfrak{F}$ if $X$ contains $\operatorname{sp}(T)$ and if $\|f(T)\| \leqq\|f\|_{X}$ for all $f$ in the set of rational functions with poles off $X$. Here, $\|f\|_{X}$ denotes the usual supremum norm of $f$ over $X$. It is easy to see that the spectrum of a subnormal operator is a spectral set. A theorem of von Neumann states that if the spectrum of any operator $A$ is a spectral set and if $R(\operatorname{sp}(A))=C(\operatorname{sp}(A))$, then $A$ must be normal; for an elegant proof see Lebow [8]. (Here, for any compact set $X, R(X)$ denotes the set of functions on $X$ which are uniformly approximable by rational functions with poles off $X$, and $C(X)$ is the set of continuous functions on $X$.) A local version of von Neumann's result for completely subnormal operators is contained in the following

Received by the editors April 6, 1971.

AMS 1970 subject classifications. Primary 47B20; Secondary 47A10.

Key words and phrases. Subnormal operators, hyponormal operators, spectrum, spectral sets, approximation by continuous functions, approximation by rational functions.

(1) This work was supported by National Science Foundation research grants.

Copyright (C) 1972, American Mathematical Society 
THEOREM. A necessary and sufficient condition that a compact set $X$ be the spectrum of a completely subnormal operator is that, for every open disk $D$,

$$
X \cap D \neq \varnothing \text { implies } R(X \cap \bar{D}) \neq C(X \cap \bar{D}),
$$

where $\bar{D}$ denotes the closure of $D$.

It was shown in Putnam [9] that if $T$ is completely hyponormal then, for any open disk $D$,

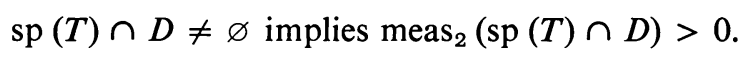

Whether the converse is true is not known. Thus, if $X$ is any compact set for which

$$
X \cap D \neq \varnothing \text { implies } \text { meas }_{2}(X \cap D)>0,
$$

does there exist a completely hyponormal $T$ for which $\operatorname{sp}(T)=X$ ? Certain evidence obtained in Clancey and Putnam [2] and in Putnam [11] suggests an affirmative answer. If this is indeed the case then it is seen that sets of measure zero play the same role for hyponormal operators as those (necessarily nowhere dense) compact sets $X$ satisfying $R(X)=C(X)$ play for subnormal operators. (In any case, the necessity portion of the above theorem has its analogue in the hyponormal case.)

It may be noted that $R(X)=C(X)$ holds for "most" nowhere dense compact sets $X$, in particular, those whose complement consists of a finite number of components. This is a consequence of a theorem of Mergelyan; cf. Rudin [12, $\mathrm{p}$. 390]. (It was shown in Putnam [10] that if $A$ is subnormal and if its spectrum is contained in a simple closed curve then $A$ is normal. This is of course a special case of the necessity part of the theorem of the present paper.) On the other hand, there exist nowhere dense compact sets for which (1.1) holds, e.g., any Swiss cheese; cf. Gamelin [3, p. 25], or Zalcman [14, p. 69]. Conditions on a compact set $X$ involving a certain type of analytic capacity and which are equivalent to the requirement that $R(X)=A(X)$ have been obtained by Vitushkin; see [3] and [14, pp. $100 \mathrm{ff}$ ]. (Here, $A(X)$ denotes the functions continuous on $X$ and analytic on its interior. If $X$ is nowhere dense, then $A(X)=C(X)$.) In particular, most of the known geometric conditions on $X$ sufficient for $R(X)=A(X)$ can be deduced from Vitushkin's theorem; see [14, pp. $112 \mathrm{ff}]$.

The proof of the theorem will be given in $\S \S 2$ and 3, and will avoid an appeal to von Neumann's theorem on spectral sets.

2. Proof of necessity. It will be shown that if $A$ is subnormal on $\mathfrak{S}$ and if, for some open disk $D$,

$$
\operatorname{sp}(A) \cap D \neq \varnothing \quad \text { and } \quad R(\operatorname{sp}(A) \cap \bar{D})=C(\operatorname{sp}(A) \cap \bar{D}),
$$

then necessarily $A$ has a reducing subspace on which it is normal. Let $A$ have the minimal normal extension $N=\int z d G_{z}$ acting on the Hilbert space $\mathfrak{T} \supset \mathfrak{S}$. Let $\mathfrak{S}_{1} \subset \mathfrak{N}$ be defined by

$$
\mathfrak{S}_{1}=G(\operatorname{sp}(A) \cap D) \mathfrak{S}_{\text {. }}
$$


Then it will be shown that

$$
0 \neq \mathfrak{S}_{1} \subset \mathfrak{S}_{2}, \quad N \mathfrak{S}_{1} \subset \mathfrak{S}_{1} \quad \text { and } \quad N^{*} \mathfrak{S}_{1} \subset \mathfrak{S}_{1} \text {, }
$$

so that $\mathfrak{S}_{1}$ is a nontrivial subspace of $\mathfrak{S C}_{\mathfrak{Z}}$ reducing $A$ and on which $A$ is normal, and the necessity part of the theorem will be established.

That $N \mathfrak{S}_{1} \subset \mathfrak{S}_{1}$ is clear. It will next be shown that $N^{*} \mathfrak{S}_{1} \subset \mathfrak{S}_{1}$. It is clear from the second part of (2.1) that $\operatorname{sp}(A) \cap \bar{D}$ is nowhere dense and hence, in particular, sp $(A)$ has no interior in $\bar{D}$. If $N^{*} \mathfrak{S}_{1} \subset \mathfrak{S}_{1}$ does not hold then there exists some $f$ in $\mathfrak{S}_{1}$ for which $N^{*} f \notin \mathfrak{S}_{1}$ and hence there exists a vector $g \in \mathfrak{K} \ominus \mathfrak{S}_{1}$ for which

$$
\left(N^{*} f, g\right) \neq 0 .
$$

Suppose that $t \notin \operatorname{sp}(A) \cap \bar{D}$. Hence, if $t \in \bar{D}$ then $t \notin \mathrm{sp}(A)$ and, since $\operatorname{sp}(N)$ $\subset \operatorname{sp}(A)$, also $t \notin \operatorname{sp}(N)$. If $x \in \mathfrak{S}_{1}$ then clearly $x=(A-t I) y=(N-t I) y$ for some $y \in \mathfrak{S}_{1}$, and so $(N-t I)^{-1} x=y$, that is, $(N-t I)^{-1} \mathfrak{S}_{1} \subset \mathfrak{S}_{1}$. If $t$ lies outside $\bar{D}$ then $(z-t)^{-1}$ is analytic on $\bar{D}$ and there exist polynomials $\left\{p_{n}(z)\right\}, n=1,2, \ldots$, converging uniformly, as $n \rightarrow \infty$, to $(z-t)^{-1}$ on $\bar{D}$. For $x \in \mathfrak{S}_{1}, p_{n}(N) x \in \mathfrak{S}_{1}$ and clearly, as $n \rightarrow \infty, p_{n}(N) x$ converges strongly to $\int_{\operatorname{sp}(A) \cap D}(z-t)^{-1} d G_{z} x$, so that this last vector is also in $\mathfrak{S}_{1}$. Consequently,

$$
\int_{\mathrm{sp}(A) \cap \bar{D}} F(z) d\left(G_{z} f, g\right)=0
$$

for $F(z)=(z-t)^{-1}$ whenever $t \notin \mathrm{sp}(A) \cap \bar{D}$. This implies that (2.5) holds for any $F \in R(\operatorname{sp}(A) \cap \bar{D})$ and hence, by $(2.1)$, for any continuous $F$ on $\operatorname{sp}(A) \cap \bar{D}$. If $F(z)=\bar{z}$, it is seen that $\left(N^{*} f, g\right)=0$, in contradiction to (2.4). This establishes the second part of (2.3).

The argument of the last paragraph is similar to that of Wermer [13] who considered the case where $\operatorname{sp}(A)$ was of measure 0 . The condition $R(\operatorname{sp}(A))$ $=C(\operatorname{sp}(A))$ was assured by a result of Hartogs and Rosenthal (cf. Gamelin [3, p. 47]).

That $\mathfrak{S}_{1} \neq 0$ is clear from the fact that $N$ is the minimal normal extension of $A$. Thus, in order to prove (2.3), there remains only to be shown that $\mathfrak{H}_{1} \subset \mathfrak{S}$. To this end, let $\alpha$ denote the closed annulus with hole $D$ and with outer radius so large that $\alpha$ includes that part of sp $(A)$ lying outside $D$. Let $Y=\alpha \cup\{\operatorname{sp}(A) \cap \bar{D}\}$. By a result of Bishop (see [3, p. 51] or [14, p. 124]), $f$ belongs to $R(Y)$ whenever $f$ is "locally" in $R(Y)$. From this it follows (see [3, p. 51]) that if

$$
F \text { is continuous on } Y \text { and } F=0 \text { on } \alpha(F),
$$

where $\alpha(F)$ denotes some open annulus (depending on $F$ ) containing $\alpha$, then $F \in R(Y)$. Thus, $F(N)=\int F(z) d G_{z}$ leaves $\mathfrak{S}$ invariant. Hence, if $f \in \mathfrak{S}$ and $g \in \mathfrak{R} \ominus \mathfrak{H}$, then (2.5) holds, where now $F$ is any function satisfying (2.6). If one chooses a uniformly bounded sequence of functions $F=F_{n}(n=1,2, \ldots)$ so as to satisfy (2.6) and so as to converge pointwise to the characteristic function of 
$\operatorname{sp}(A) \cap D$, it follows from (2.5) and a term by term integration theorem of Lebesgue that $(G(\operatorname{sp}(A) \cap D) f, g)=0$, that is, $\mathfrak{S}_{1} \subset \mathfrak{S}_{\text {. }}$. This completes the proof of the necessity portion of the theorem.

3. Proof of sufficiency. Suppose that $X$ is a compact set for which (1.1) holds for any open disk $D$. It will be shown that there exists a completely subnormal operator $A$ for which $X=\operatorname{sp}(A)$.

First, suppose that $X$ is the closure of its interior $X_{0}$. Let $\mathfrak{S}=\mathfrak{S}_{\mathfrak{C}}\left(X_{0}\right)$ denote the Hilbert space of functions analytic on $X_{0}$ and square integrable with respect to Lebesgue planar measure, and let $A$ denote the multiplication operator $A=z$ on S. (See Halmos, Lumer and Schaeffer [7] for details.) Then $A$ is subnormal and, in fact, completely subnormal. In fact, otherwise, there would exist some $f \neq 0$ in $\mathfrak{F}$ for which both $z f$ and $\bar{z} f$ belong to $\mathfrak{F}$, and this is clearly impossible.

Since the spectrum of the direct sum of two operators is simply the union of the two spectra, and since any compact set $X$ can be expressed as the union of the closure of its interior and a nowhere dense compact set, it is clearly enough to prove the sufficiency part of the theorem in case $X$ has no interior.

Recall that a point $x$ in a compact set $Y$ is a peak point for $R(Y)$ if there exists an $f$ in $R(Y)$ satisfying $f(x)=1$ and $|f(y)|<1$ for $y$ in $Y, y \neq x$. A theorem of Bishop states that $R(Y)=C(Y)$ if and only if every point of $Y$ is a peak point of $R(Y)$; cf. [3, p. 54]. A measure $\mu$ is called a representing measure at $x$ in $R(Y)$ in case $\mu$ is a Baire probability measure on $Y$ satisfying

$$
f(x)=\int_{Y} f d \mu, \quad f \in R(Y) .
$$

A point $x$ in $Y$ is a peak point if and only if the only representing measure at $x$ for $R(Y)$ is the point mass measure (that is, a unit mass concentrated at $x$ ); cf. [14, p. 5].

As noted above, it can now be assumed that $X$ has no interior. It follows from (1.1) and from Bishop's result, that we can choose a countable set of points $\left\{x_{n}\right\}$, $n=1,2, \ldots$, which are not peak points of $R(X)$, and for which $\left\{x_{n}\right\}$ is dense in $X$. Let $\mu_{n}$ be any nonpoint mass representing measure at $x_{n}$ for $R(X)$ and let $\nu_{n}$ $=\left(z-x_{n}\right) \mu_{n}$. Let $\left|\nu_{n}\right|$ denote the total variation measure of $\nu_{n}$ (see Rudin [12, p. 126]); thus there exists a $\left|\nu_{n}\right|$-measurable function $h_{n}=h_{n}(x)$ on $X$ satisfying

$$
\nu_{n}=h_{n}\left|\nu_{n}\right|, \quad\left|h_{n}\right|=1 \text {. }
$$

Let $R^{2}\left(d\left|\nu_{n}\right|\right)$ denote the subspace of $L^{2}\left(d\left|\nu_{n}\right|\right)$ spanned by $R(X)$. Then, for $f \in R(X)$, $\int_{X} f h_{n} d\left|\nu_{n}\right|=\int_{X} f\left(z-x_{n}\right) d \mu_{n}=0$, so that

$$
\bar{h}_{n} \perp R^{2}\left(d\left|\nu_{n}\right|\right), \quad n=1,2, \ldots
$$

If $A_{n}$ is the multiplication operator $z$ on $R^{2}\left(d\left|\nu_{n}\right|\right)$ then $A_{n}$ is subnormal. It will be shown that

$$
x_{n} \in \operatorname{sp}\left(A_{n}\right)
$$


It is easy to see that the (minimal) normal extension of $A_{n}$ is the multiplication operator $B_{n}=z$ on $L^{2}\left(d\left|v_{n}\right|\right)$, and so (Halmos [5]) $\operatorname{sp}\left(B_{n}\right) \subset \operatorname{sp}\left(A_{n}\right)$. In order to prove (3.4) then, it is sufficient to show that $x_{n} \in \mathrm{sp}\left(B_{n}\right)$, that is, that every neighborhood of $x_{n}$ has positive $\left|\nu_{n}\right|$ measure. But if some neighborhood $N$ of $x_{n}$ has zero $\left|\nu_{n}\right|$ measure, then, for $y \notin X$,

$$
0=\int_{X-N}(z-y)^{-1}\left(z-x_{n}\right) d \mu_{n}=\mu_{n}(X-N)+\left(y-x_{n}\right) \int_{X-N}(z-y)^{-1} d \mu_{n} .
$$

Hence, on letting $y \rightarrow x_{n}(y \notin X)$, one obtains $\mu_{n}(X-N)=0$, a contradiction, since $\mu_{n}$ is not a point measure at $x_{n}$. This proves (3.4).

Next, let $A=\sum \oplus A_{n}$ on $\mathfrak{S}=\sum \oplus R^{2}\left(d\left|\nu_{n}\right|\right)$. Since $\mu_{n}$, hence $\left|\nu_{n}\right|$, has support on $X$, then $\operatorname{sp}\left(A_{n}\right) \subset X$, hence $\operatorname{sp}(A) \subset X$. This follows from the fact that each $A_{n}$ is subnormal. For if $t \notin X$, then $\left\|\left(A_{n}-t I\right) f_{n}\right\| \geqq\left\|\left(A_{n}^{*}-\bar{t} I\right) f_{n}\right\| \geqq \operatorname{dist}(t, X)\left\|f_{n}\right\|$ for each $f_{n} \in R^{2}\left(d\left|\nu_{n}\right|\right)$ and hence $\|(A-t I) f\| \geqq\left\|\left(A^{*}-\bar{t} I\right) f\right\| \geqq \operatorname{dist}(t, X)\|f\|$ for $f \in \mathfrak{S}=\sum \oplus R^{2}\left(d\left|\nu_{n}\right|\right)$. On the other hand, it follows from (3.4) and the fact that the $\left\{x_{n}\right\}$ are dense in $X$ that $X \subset \operatorname{sp}(A)$. Thus $\operatorname{sp}(A)=X$.

There remains to be shown that $A$ is completely subnormal, that is, that each $A_{n}$ on $R^{2}\left(d\left|\nu_{n}\right|\right)$ is completely subnormal. Suppose that, for a fixed $n, \mathfrak{M}$ is a subspace of $R^{2}\left(d\left|v_{n}\right|\right)$ which reduces $A_{n}$ and on which $A_{n}$ is normal. If $m$ is in $\mathfrak{M}$, then so are $z m$ and $\bar{z} m$. Hence, for any continuous $g$ on $X, g m \in \mathfrak{M}$, and so, by (3.3), $\int_{X} g m h_{n} d\left|\nu_{n}\right|=0$. Since the continuous functions on $X$ are dense in $L^{2}\left(d\left|\nu_{n}\right|\right)$, it follows that $m h_{n}=0$ a.e. with respect to $\left|v_{n}\right|$, and hence, since $\left|h_{n}\right|=1, m=0$ a.e. Thus $\mathfrak{M}=0$, and so $A_{n}$ is completely subnormal.

ACKnowledgment. The authors are indebted to A. Davie and J. Garnett for suggesting the use of the nonpoint mass representing measures in this section.

\section{REFERENCES}

1. J. Bram, Subnormal operators, Duke Math. J. 22 (1955), 75-94. MR 16, 835.

2. K. F. Clancey and C. R. Putnam, The spectra of hyponormal integral operators, Comment. Math. Helv. (to appear).

3. T. W. Gamelin, Uniform algebras, Prentice-Hall, Englewood Cliffs, N. J., 1969.

4. P. R. Halmos, Normal dilations and extensions of operators, Summa Brasil. Math. 2 (1950), 125-134. MR 13, 359.

5. - - Spectra and spectral manifolds, Ann. Soc. Polon. Math. 25 (1952), 43-49. MR 14, 1096.

6. —_, A Hilbert space problem book, Van Nostrand, Princeton, N. J., 1967. MR 34 \#8178.

7. P. R. Halmos, G. Lumer and J. J. Schäffer, Square roots of operators, Proc. Amer. Math. Soc. 4 (1953), 142-149. MR 14, 767.

8. A. Lebow, On von Neumann's theory of spectral sets, J. Math. Anal. Appl. 7 (1963), 64-90. MR 27 \#6149.

9. C. R. Putnam, An inequality for the area of hyponormal spectra, Math. Z. 116 (1970), 323-330.

10. - The spectra of subnormal operators, Proc. Amer. Math. Soc. 28 (1971), 473-477.

11. - The spectra of completely hyponormal operators, Amer. J. Math. (to appear). 
12. W. Rudin, Real and complex analysis, McGraw-Hill, New York, 1966. MR 35 \#1420.

13. J. Wermer, Report on subnormal operators, Report of an International Conference on Operator Theory and Group Representations, Arden House, Harriman, N. Y., 1955, pp. 1-3; Publ. \#387, National Academy of Sciences-National Research Council, Washington, D.C., 1955. MR 17, 880.

14. L. Zalcman, Analytic capacity and rational approximation, Lecture Notes in Math., no. 50, Springer-Verlag, Berlin and New York, 1968. MR 37 \#3018.

Department of Mathematics, University of California, Los Angeles, California 90024

Department of Mathematics, Purdue University, Lafayette, Indiana 47907

Current address (Clancey): Department of Mathematics, University of Georgia, Athens, Georgia 30601 